\title{
RADIATION FROM PROBE SURFACES BOMBARDED BY ELECTRONS
}

\author{
By F. L. Mohler and C. Boeckner
}

ABSTRACT

Metals in the form of small probe surfaces are held at a positive potential in the highly ionized region of a discharge (commonly a cæsium discharge) and under intense electron bombardment emit radiation. Photographic spectrophotometric measurements are made. Radiations at 7 volts from $\mathrm{W}, \mathrm{Al}, \mathrm{Be}$, Au alloyed with $\mathrm{Cs}$, Th, and Pt are similar in intensity and intensity distribution. The spectral energy distribution on a wave-length scale is nearly constant between 6,400 and $2,400 \mathrm{~A}$ with a radiation flux of about $20 \mathrm{ergs}$ for a wave-length range of $1 \mu$ and for a current density of 1 ampere per $\mathrm{cm}^{2}$. $J(\lambda)$ for $\mathrm{Cu}$ has a pronounced increase in the red and for silver a tenfold increase between 3,200 and $3,600 \mathrm{~A}$. Above 6 volts this intensity distribution remains the same while with lower voltages it drops almost linearly to zero with increasing $\nu$. The threshold value of $\nu$ agrees with the relation $h \nu=e(V+W)$ where $W$ is 1.45 volts for tungsten and about 2 volts for $\mathrm{Cu}, \mathrm{Ag}$, and $\mathrm{Al}$. The voltage variation of intensity is different for each metal. The intensity and intensity distribution of tungsten and silver radiation in a helium discharge are similar to values obtained in cæsium vapor.

The equal energy spectrum common to all metals is probably analogous to continuous $\mathrm{X}$ rays. The selective radiation of silver and copper may correspond to characteristic $\mathrm{X}$ radiation. The high-frequency edge of the silver emission band has the shape of a Fermi distribution of electrons at $370^{\circ} \mathrm{C}$., suggesting that the emission process involves the ionization of an underlying level and the fall of a conduction electron into that level.

\section{CONTENTS}

Page

I. Method and procedure

II. Intensity distribution at 7 volts

1. Tungsten

2. Aluminum

3. Beryllium

4. Gold-cæsium alloy

5. Thorium

6. Platinum

7. Copper........

8. Silver

III. Intensity distribution at other voltages.

IV. Intensity as a function of voltage

V. Probe radiation in a helium discharge

VI. Discussion

1. The equal energy spectrum

2. Selective radiation of silver and copper

3. The variation of intensity with voltage
751

753

753

753

753

754

754

754

754

755

755

756

758

759

759

760

763

\section{METHOD AND PROCEDURE}

An earlier paper describes measurements of a continuous spectrum emitted by metals under low voltage electron bombardment. ${ }^{1}$ The large currents required to make the radiation measurable were obtained by the device of using the metal in the form of a small

1 Mohler, F. L., and Boeckner, C., B. S. Jour. Research, vol. 6 (RP297), p. 673 April, 1931. Referred to as "first paper." 
probe surface in the highly ionized region of a fairly intense discharge. Measurements by the same method have been extended to other metals and to a larger voltage range, and this paper reports the new results.

In Figure 1 we repeat from the first paper a diagram of the type of tube used. A discharge current of about an ampere is maintained between the hairpin cathode and the cylindrical anode while the probe surface, which is the end of a heavy wire about $2 \mathrm{~mm}$. in diameter, is held at a potential positive with respect to the surrounding space. The current density at the probe surface was commonly from 2 to 5 amperes per $\mathrm{cm}^{2}$. From the current voltage characteristics of the probe, the voltage drop between the probe and the surrounding space and the average energy of the electrons in the space can be determined. This energy, about 0.4 electron volts, added to the energy derived from the voltage drop gives the average energy

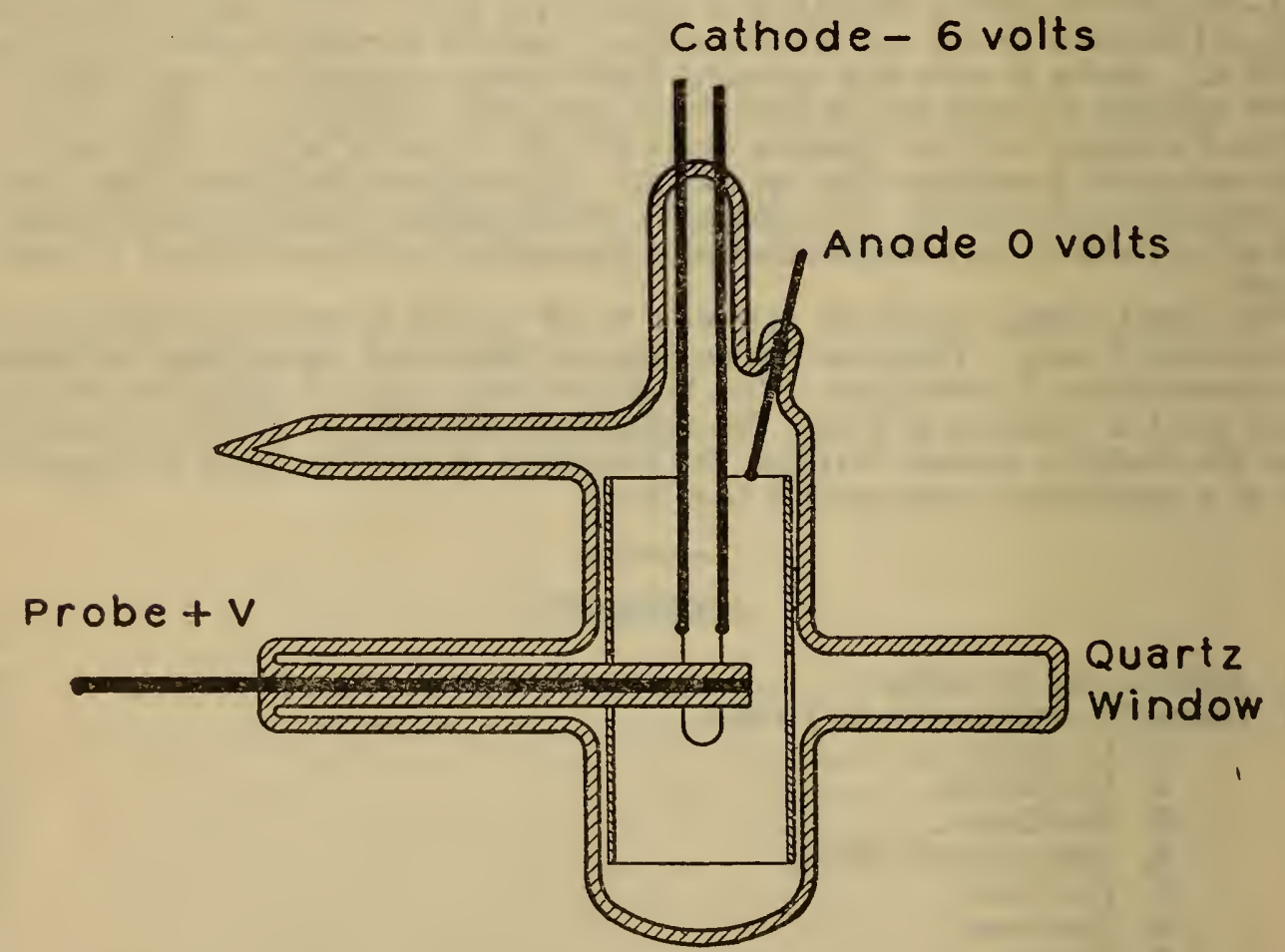

FIGURE 1.-Type of tube used for observation of probe radiation

of the electrons striking the surface. The corrected potential is referred to as the applied potential in this paper.

Spectra have been photographed with a Hilger $E_{2}$ quartz spectrograph, a small quartz spectrograph, and a 3-prism Zeiss glass spectrograph. Exposure times ranged from 15 minutes to many hours. For comparison of metals two probes have been mounted side by side in the discharge.

Relative intensity measurements were based on densitometer comparisons of two or more exposures with full light and light reduced in a known ratio and one or more exposures under different conditions of intermediate density. Intensity-distribution measurements are based on comparison with spectra of a calibrated tungsten strip lamp in quartz. A correction for failure of the reciprocity law has been applied where exposure times differ, and some results of the first paper have been recomputed on this basis. 
An obvious difficulty of the experiments is that the faint continuous spectrum must be viewed in the presence of a brilliant discharge. The cæsium discharge gives the least trouble in this respect and so has been used in most of this work, although conditions of metal surfaces in cæsium vapor are complicated by the fact that a monatomic layer of cæsium is occluded on most metals. With intermediate voltages the ultra-violet spectrum appeared to be free from scattered light except for isolated emission lines. Under other conditions there was a more or less continuous background which could be partially corrected for by making a blank exposure with the probe current off, but since the light in front of the probe is intensified when it is drawing a current, measurements become unreliable when the background correction is large.

Contamination of the probe surface from sputtering or evaporation from the cathode or from a neighboring probe was a serious source of error. The surface could usually be brought back to its initial state by bombarding it with positive ions at -100 volts for a few minutes. Many metals combine with cæsium, but there was no evidence of combination with any of the metals here used except gold which forms a silvery white alloy. The statement in the first paper that silver appeared to combine with cæsium is now judged to be erroneous.

It has been shown in the first paper that the probe radiation is independent of vapor pressure and other discharge conditions and that the intensity is proportional to the probe current. The significant variables to be investigated are the intensity as a function of wave length and voltage.

\section{INTENSITY DISTRIBUTION AT 7 VOLTS}

The intensity of radiation from tungsten at 7 volts has been taken as a standard-perhaps unfortunately, as there are inconsistencies of the order of twofold between some comparative measurements as compared with direct measurements obtained with the strip lamp. The absolute value here given is about half the value given in the first paper, and is probably uncertain by a factor of 2 .

\section{TUNGSTEN}

Figure 2 gives the intensity distribution of tungsten radiation based on four overlapping series of measurements which show that between 2,600 and $5,200 \AA$ the intensity per unit wave length range, $J(\lambda)$, is nearly constant. The absolute value as given by the mean of the ultra-violet measurements is 20 ergs per $\mathrm{cm}^{2}$ per sec. for a wave-length range of $1 \mu$ and a current density of 1 ampere per $\mathrm{cm}^{2}$.

\section{ALUMINUM}

Comparative measurements with tungsten show that from 4,900 to $2,300 \mathrm{~A}$ the intensity per unit current is almost identical with tungsten, while comparison with a strip lamp shows that $J(\lambda)$ remains nearly constant to $6,400 \mathrm{~A}$.

\section{BERYLLIUM}

The intensity and intensity distribution are about the same as for tungsten. 


\section{GOLD-CAESIUM ALLOY. (Silvery in appearance)}

The intensity and intensity distribution also resemble tungsten.

\section{THORIUM}

Thorium radiation is about twice as bright as tungsten with the same intensity distribution.

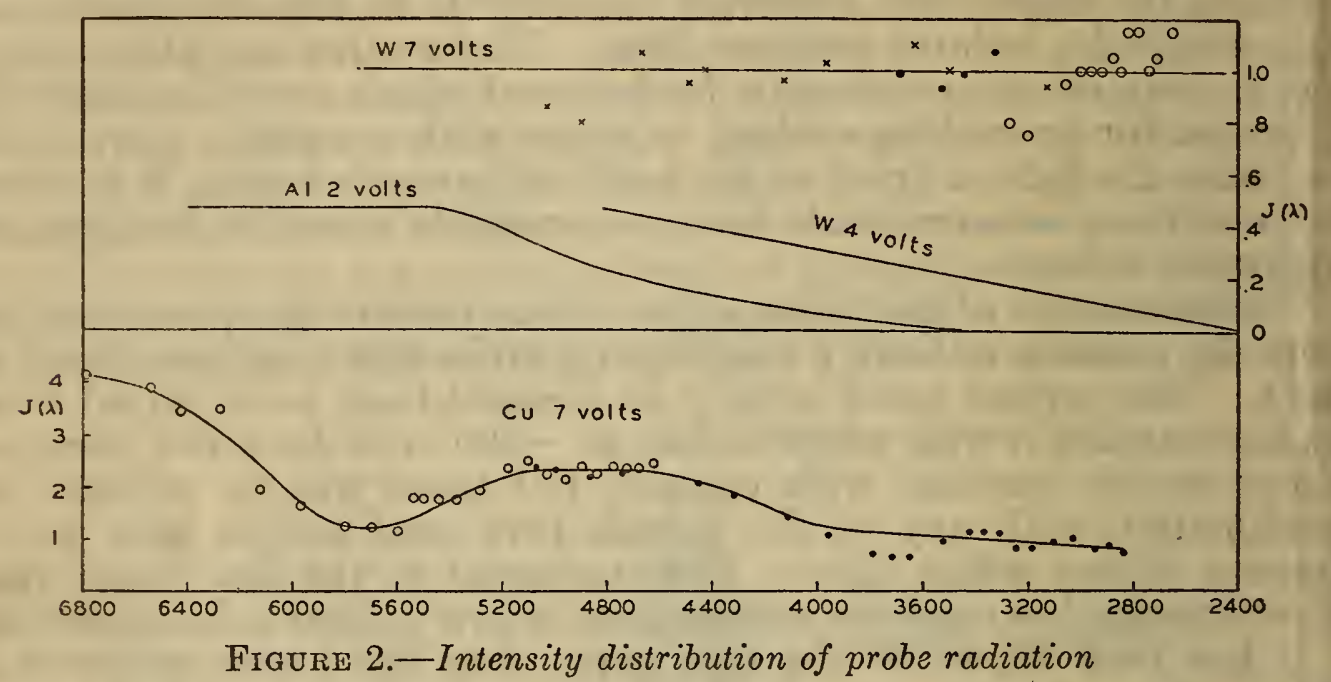

All curves are on a scale of $J(\lambda)$ for tungsten at 7 voits as unity with the unit equal approximately to 20 ergs per $\mathrm{cm}^{2}$ for a current of one ampere per $\mathrm{cm}{ }^{2}$.

\section{PLATINUM}

For platinum $J(\lambda)$ dropped regularly from 1.4 times the tungsten intensity at $3,700 \mathrm{~A}$ to 0.7 times tungsten at $2,400 \mathrm{~A}$.

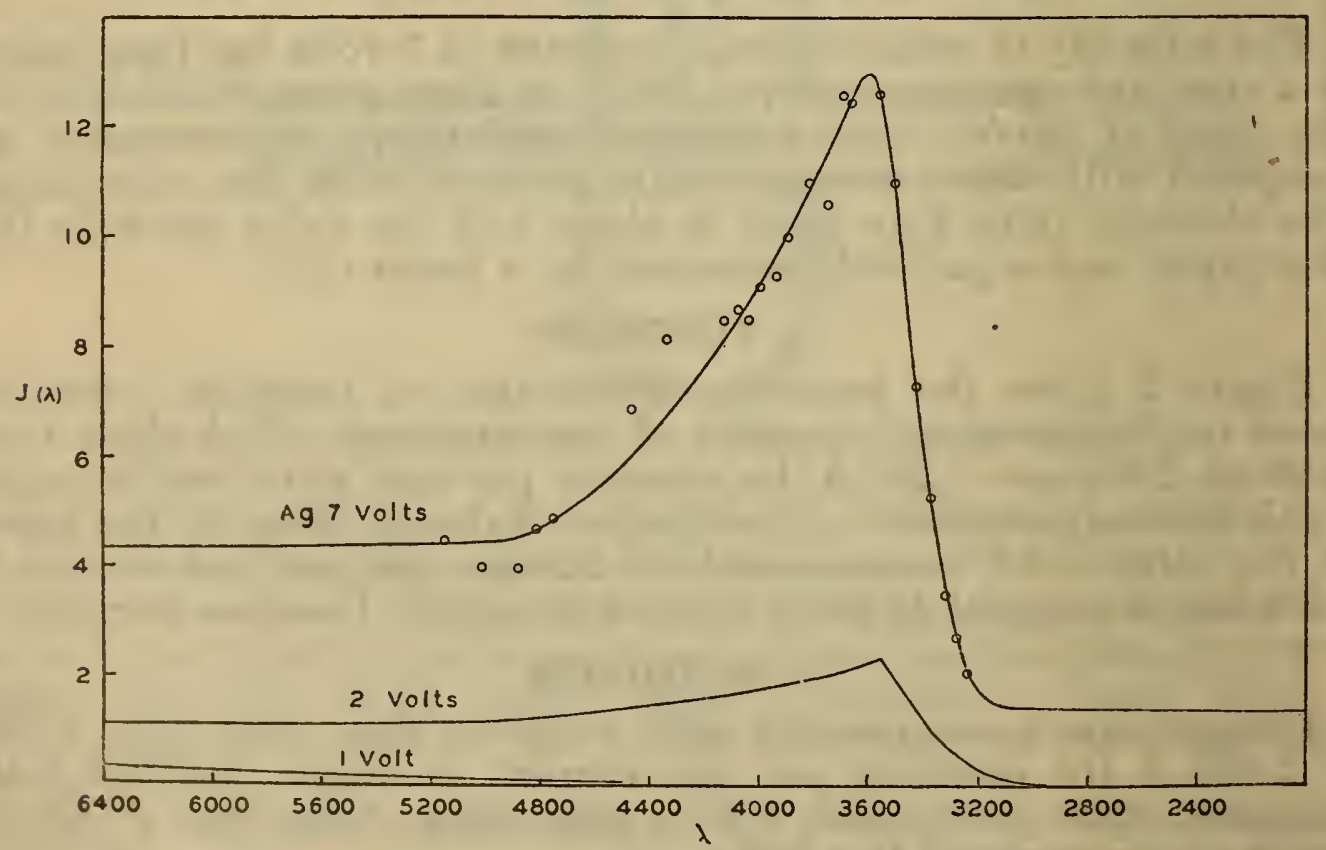

FIGURE 3.-Intensity distribution of silver radiation with ordinates in units of Figure 2

\section{COPPER}

Data in the first paper have been recomputed to include factors neglected before and results are plotted in Figure 2. The intensity for wave lengths shorter than $3,600 \mathrm{~A}$ is about the same as for tung- 
:ten; for longer wave lengths there are marked differences. The sxperimental uncertainty becomes extreme at the red end of the urve.

\section{SILVER}

The intensity distribution for silver radiation is in marked contrast to other metals. Several sets of data have been averaged to give the sxtreme portions of the curve in Figure 3 while a single set has been used near the maximum to avoid a false rounding of the sharp maximum. Ordinates are on the same scale as Figure 2, viz, $J(\lambda)$ for tungsten equal to unity. $J(\lambda)$ for wave lengths less than $3,200 \mathrm{~A}$ is much the same as for other metals.

\section{INTENSITY DISTRIBUTION AT OTHER VOLTAGES}

The intensity changes with the voltage in a manner described in the following section, but, in general, the intensity distribution remains nearly the same as the potential is increased above 7 volts: Below 6 or 7 volts $J(\lambda)$ decreases with decreasing wave length and for most metals with applied voltages below 4 volts $J(\lambda)$ approaches zero in the range of the quartz spectrograph.

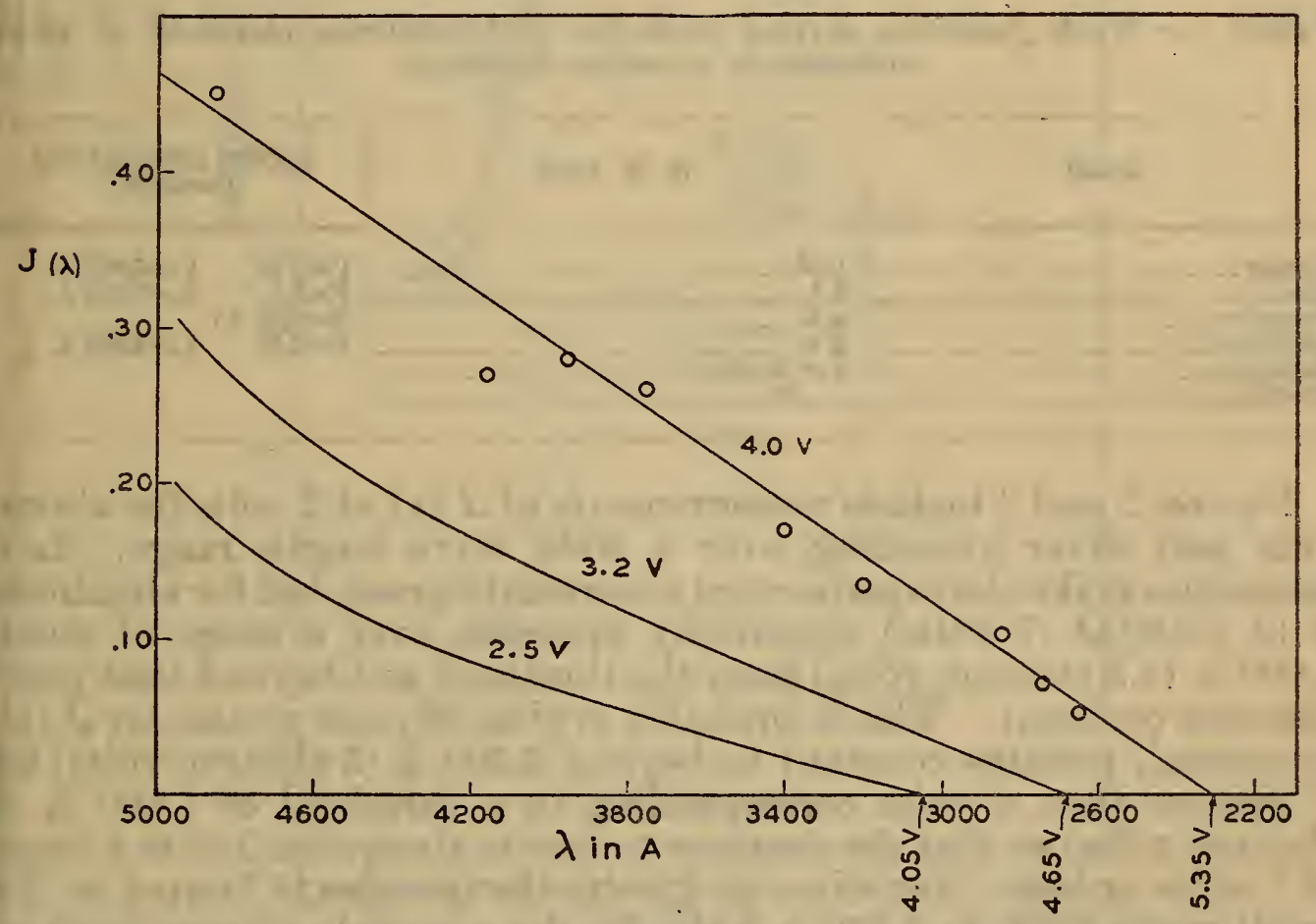

FIGURE 4.-Intensity distribution of tungsten radiation at low voltage in units of Figure 2

Below each intercepi is given the voltage corresponding to the threshold wave length.

Figure 4 gives the intensity distribution of tungsten spectra at $2.5,3.2$, and 4.0 volts on a scale of $J(\lambda)$ at 7 volts $=1$. As $J(\lambda)$ is nearly a linear function of $\lambda$ the extrapolation to zero intensity gives a definite and reproducible value for the high-frequency threshold. The values of these high-frequency thresholds are given in terms of $h \nu / e$ in volts. The three values satisfy the equation.

$$
h \nu=e(V+W) \quad W=1.45 \pm .1 \text { volts }
$$


where $V$ is the applied voltage and $W$ is interpreted to be the work function of the cæasiated metal. In the first paper we report an independent series of measurements leading to an identical value of $W$.

Figure 3 of the first paper and Figures 2 and 3 of this paper include plots of $J(\lambda)$ at low voltages for copper, silver and aluminum as well as for tungsten and it is seen that $J(\lambda)$ approaches zero in a similar manner for all these metals. For thorium and platinum the intensity distribution at 3 volts is practically the same as at 7 volts with no evidence of a threshold in the range of the quartz spectrograph. We conclude that the work function is greater than 3 volts which would give a threshold near 2,100 A. Table 1 summarizes the values of the work functions derived from this work. The values of 2.1 volts or less are of the magnitude to be expected for cæsiated metals, but there is no safe basis for quantitative comparison. Thus, Ives and Olpin ${ }^{2}$ find that the photo-electric threshold depends critically on the thickness of the alkali film on a metal and very little on the nature of the underlying metal. For cæsium on silver and platinum the minimum value of the work function was 1.38 volts. It is consistent with their conclusions that all our observed values are somewhat greater than this.

TABLE 1.-Work functions derived from the high-frequency threshold of probe radiation in a cæsium discharge

\begin{tabular}{|c|c|c|}
\hline Metal & $W$ in volts & $\begin{array}{l}\text { Applied potential and } \\
\text { threshold }\end{array}$ \\
\hline $\begin{array}{l}\text { Copper } \\
\text { Silver } \\
\text { Tungsten } \\
\text { Aluminum } \\
\text { Platinum } \\
\text { Thorium }\end{array}$ & $\begin{array}{l}1.95 \\
1.45 \\
2.10 \\
3 \text { or more }\end{array}$ & $\begin{array}{ll}V=2.15 . & \lambda_{0}=3,000 \mathrm{~A} \\
V=2.2 . & \lambda_{0}=2,860 \mathrm{~A} \\
\text { (See fig. 4.) } & \\
V=2.55 . & \lambda_{\bullet}=2,650 \mathrm{~A}\end{array}$ \\
\hline
\end{tabular}

Figures 2 and 3 include measurements of $J(\lambda)$ at 2 volts for aluminum and silver extending over a wide wave length range. Low intensities make the experimental uncertainty great, but for aluminum $J$ (2 volts)/J (7 volts) apparently increases over a range of about $2,000 \mathrm{~A}$ (1.3 electron volts) from the threshold and beyond that point becomes constant. This is probably typical of most metals for $J(\lambda)$, in general, remains constant to beyond $2,500 \mathrm{~A}$ ( 5 electron volts) for $V$ greater than 6 volts corresponding to a threshold of $1,600 \mathrm{~A}$ ( 8 electron volts) so that the decrease to zero is always limited to a range of 3 volts or less. For silver at 2 volts the increase is limited to the small range 2,800 to $3,200 \mathrm{~A}$ while for the relatively intense band on the red side of $3,200, J$ ( 2 volts $) / J$ (7 volts) remains constant.

\section{INTENSITY AS A FUNCTION OF VOLTAGE}

The foregoing results have shown a marked similarity between most metals, but the voltage variation of intensity is strikingly characteristic of the metal used. A comparison of the intensities at one wave length and different voltages is intrinsically simpler than a

Ives and Olpin, Phys. Rev., vol. 34, p. 117; 1929. Ives, Astrophys. J., vol. 60, p. 4; 1924. 
measurement of intensity distribution. Measurements could be reproduced to about 5 per cent though occasionally much greater rariations were caused by contamination of the surfaces. Figures 5 and 6 give the results. Data for tungsten, silver, and aluminum were checked by repeated series of measurements. (The tungsten point at 10 volts is probably in error because of surface contamina-

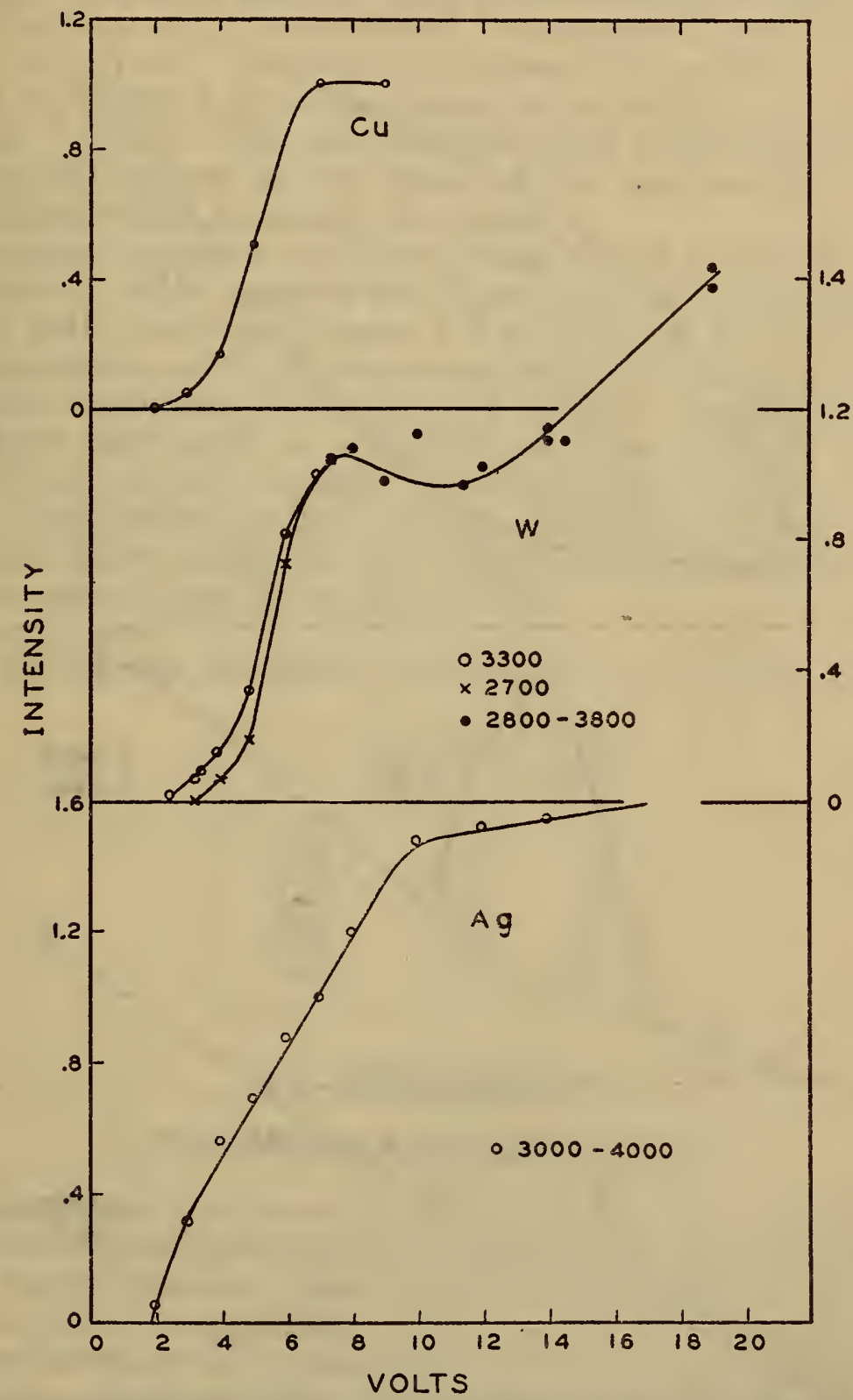

FIgURe 5.-Voltage variation of monochromatic intensity

The ordinate at 7 volts on each curve is taken as 1.

tion.) In general, the intensity distribution remains the same within about 10 per cent for voltages above 5 volts and the plotted points are a mean of measurements at many wave lengths between 2,600 and 4,000 A where other wave lengths are not specified. The voltage threshold and the slope of the curve near the threshold depend on the wave length as described in the preceding section. 


\section{PROBE RADIATION IN A HELIUM DISCHARGE}

Some effort has been made to obtain measurements of radiation from probe surfaces in a helium discharge as such radiation could more safely be assumed to be characteristic of a pure metal. Scattered

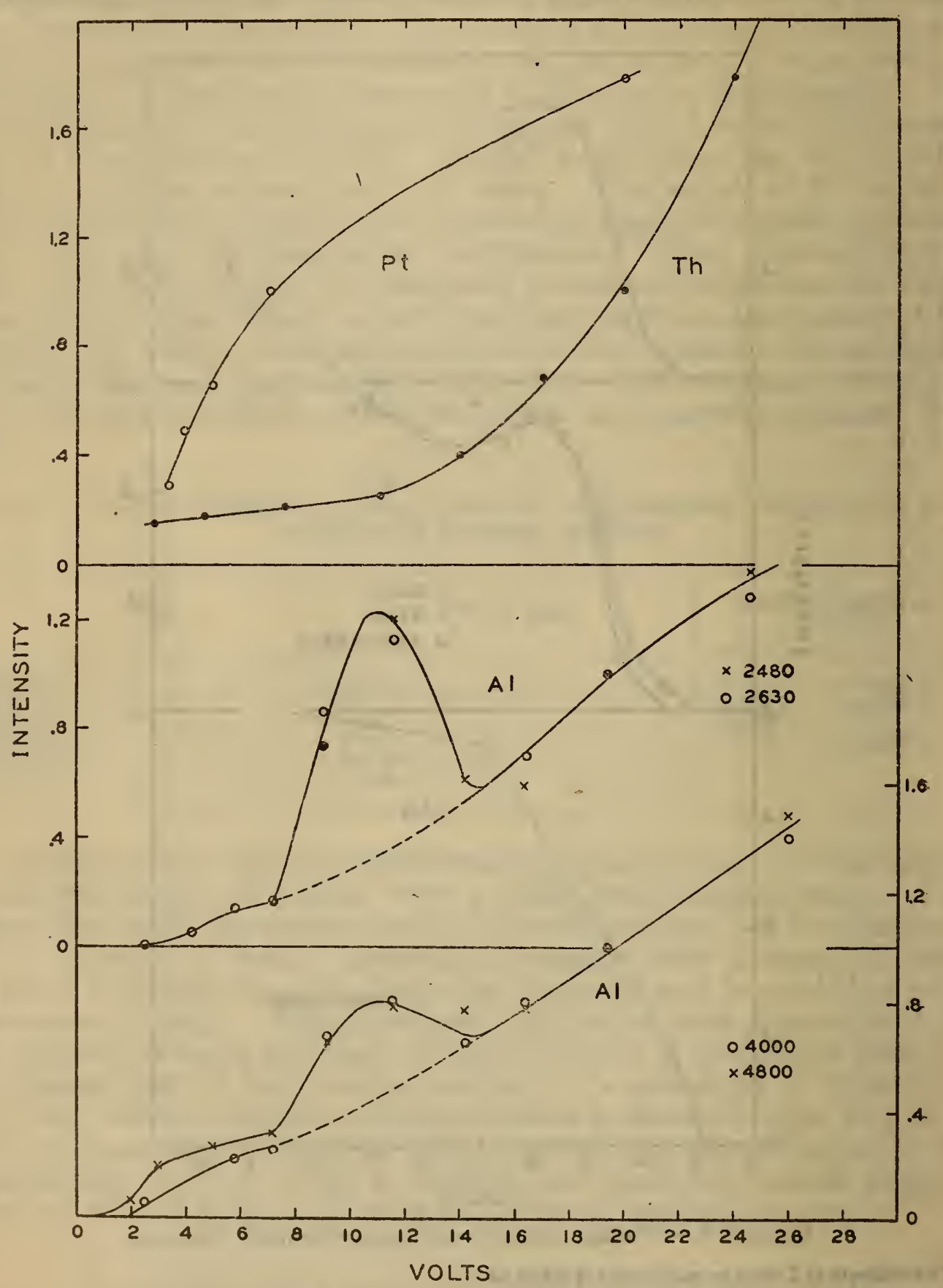

FIGURE 6.-Voliage variation of monochromatic intensity

light was usually very much greater than in the cæsium discharge and it was impossible to maintain steady conditions over a long period of time. Because of the high-ionization potential of helium higher probe potentials could be used and were used to increase the intensity and reduce errors from scattered light. 
A comparison between radiation from tungsten in a helium discharge at 21 volts and radiation from a similar probe in cæsium at 7 volts gives the following ratio of intensity per unit current. $J$ (helium) $/ J$ (cæsium) at $3,270 \mathrm{~A}, 3.2 ; 3,100 \mathrm{~A}, 3.5 ; 2,900 \mathrm{~A}, 4.1 ; 2,680 \mathrm{~A}, 3.9$; $2,600 \mathrm{~A}, 4.2$; mean, 3.8. The variation of the ratio with wave length is no greater than the experimental uncertainty. Since pure tungsten has a work function 3 volts greater than cresiated tungsten, the intensity in helium at 21 volts should be compared with the intensity in cæsium at 24 volts. A rather uncertain extrapolation of the tungsten curve of Figure 5 gives the intensity at 24 volts as 1.9 times the intensity at 7 volts. The observed intensity is approximately twice what we would expect on the basis of the cæsium data while the intensity distribution is nearly the same.

A comparison between radiation from silver and tungsten could only be made at wave lengths less than $3,200 \mathrm{~A}$. The ratio $J(\mathrm{Ag}) / J$ (W) at 28 volts increased from 1.4 at $2,760 \mathrm{~A}$ to 2.4 at $3,110 \mathrm{~A}$. The ratio in cæsium at 7 volts remained about 1.5 in this range.

The silver radiation was compared with a tungsten strip lamp between 3,000 and $3,490 \mathrm{~A}$. Table 2 gives intensities at 28 volts in helium and at 7 volts in cæsium reduced to the same scale. Except for minor discrepancies which may be accidental the intensity distribution of silver radiation in helium shows the characteristics found at lower voltages in cæsium vapor.

TABLE 2.-Intensity distribution of silver radiation in cæsium and helium

\begin{tabular}{|r|r|r|}
\hline$\lambda$ & \multicolumn{1}{|c|}{$\begin{array}{c}(28 \text { volts }) \\
\text { in He }\end{array}$} & $\begin{array}{r}\mathrm{J} \text { (7 volts) } \\
\text { in Cs }\end{array}$ \\
\hline 3,000 & 1.5 & 1.4 \\
3,100 & 2.5 & 1.5 \\
3,200 & 4.6 & 2.0 \\
3,310 & 4.9 & 3.4 \\
3,390 & 6.0 & 5.6 \\
3,490 & 8.5 & 10.0 \\
\hline
\end{tabular}

\section{DISCUSSION}

\section{THE EQUAL ENERGY SPECTRUM}

It has been found that the radiation intensity and intensity distribution at 7 volts applied potential is nearly the same for all metals except silver and copper and for these as well at wave lengths shorter than $3,000 \mathrm{~A}$. An obvious possibility that it is the surface layer of cæsium which is radiating in all cases except silver and copper seems to be ruled out on several grounds. The intensity voltage curves seem to be characteristic of the metal used; the intensity of probe radiation in a helium discharge is of the same magnitude as in cæsium; the cæsium layer is probably monatomic and the incident electrons as well as the emergent light can penetrate 10 to 100 atom diameters. For these reasons it seems safe to assume that most metals give nearly identical spectra.

In the first paper we interpreted this radiation as analogous to the continuous X-ray spectrum and applied the general equation for $74280-31-10$ 
intensity of continuous X-rays from a thick target as given by Kramers. ${ }^{3}$ The radiation per unit frequency range is

$$
J(\nu)(\mathrm{ergs})=i \times 5 \times 10^{-50} Z\left(\nu_{0}-\nu\right)
$$

where $i$ is the number of electrons striking the surface per second, $Z$ is the atomic number and $\nu_{0}$ is the threshold frequency given by

$$
h \nu_{0}=e(V+W)
$$

Our results show $J$ to be quite independent of $Z$, but this is not entirely unexpected. Equation (1) involves $Z$ because high-frequency radiation is assumed to result from a transition between hyperbolic orbits in the immediate vicinity of the nucleus. For the low frequencies here considered the transition can take place much nearer the atom surface and for all elements beyond some undetermined value of $Z$ the intensity will cease to depend on $Z$. We found that for $i=0.63 \times 10^{19}$ per $\mathrm{cm}^{2}, \nu_{0}=2.2 \times 10^{15}$ ( $V+W=9$ volts), $\nu=1 \times 10^{15}$ $(3,000 \mathrm{~A}), J(\nu)$ was about $6 \times 10^{-15} \mathrm{ergs} \operatorname{per} \mathrm{cm}^{2}(J(\lambda)=20 \mathrm{ergs})$ for all metals. $J(\nu)$ by equation (1) is $3.8 \times 10^{-16} \times Z$ ergs for the above values of $i, \nu$ and $\nu_{0}$. Dividing the observed value by the coefficient of $Z, 3.8 \times 10^{-16}$, gives the value of an effective nuclear charge $Z_{e}$ of about 16. Admitting an uncertainty of at least twofold such a value is not inconsistent with observations except for beryllium for which $Z=4$. The above reasoning is extremely crude, but any adequate treatment would be very complicated.

In the first paper the continuous spectrum was also compared with theoretical predictions as to the converse of the photo-electric effect. The agreement in order of magnitude for the case of copper holds equally well for the revised values of $J(\lambda)$ and for all of the metals.

\section{SELECTIVE RADIATION OF SILVER AND COPPER}

The high maximum in silver radiation near $3,600 \mathrm{~A}$ is clearly a selective radiation superposed on the equal energy spectrum and of entirely different origin. The anomalies in copper radiation are less striking but presumably of a similar kind. For silver the increase begins at $3,200 \mathrm{~A}$ where the optical constants of silver suddenly change. Both reflection and absorption increase in the range in which the emission increases so that loss of radiation by internal reflection and absorption reduces the observed effect. A correction for loss by absorption is impossible, but a correction for reflection can be applied by dividing observed values by $1-R$ where $R$ is the reflection coefficient. For two reasons this correction is uncertain. The probe surfaces were unpolished, and the reflection for light originating close to the surface may be appreciably less than for plane waves. The over correction for reflection is in the direction to compensate for the neglect of absorption. Figure 7 shows the intensity distribution for silver and copper thus corrected for reflection. ${ }^{4}$ The form of the silver curve has been entirely changed and instead of a maximum 10 times the usual value of $J(\lambda)$ we have a nearly con-

8 Kramers, Phil. Mag., vol. 46, p. 836; 1923.

Optical Constants from I. C. T., vol. 5, p. 248, and Landoldt u. Bornstein, Ergänzungsband, vol. 1, p. 471 . 
stant intensity fifty times the usual value for wave lengths longer than 3,600 . Beyond $4,500 \mathrm{~A}, R$ is over 0.9 and the correction very large and uncertain. For all other metals in the ultra-violet $R$ is less than 0.5 and a reflection correction is unimportant compared with other uncertainties.

For comparison we include plots of the absorption of silver and copper; that is, the quantity $4 \pi n k / \lambda$ from the equation

$$
J / J_{0}=\exp (-4 \pi n k / \lambda) x
$$

It is of interest that $k$ for silver increases at exactly the same wave length and in nearly the same way as the corrected intensity distribution. Evidently the increase in copper radiation in the red is similarly related to the optical constants though the phenomena are less striking.

Houston ${ }^{5}$ has suggested that this selective radiation is analogous to a characteristic X-ray emission. The impacting electron ionizes an underlying electron level and one of the "free" conduction elec-

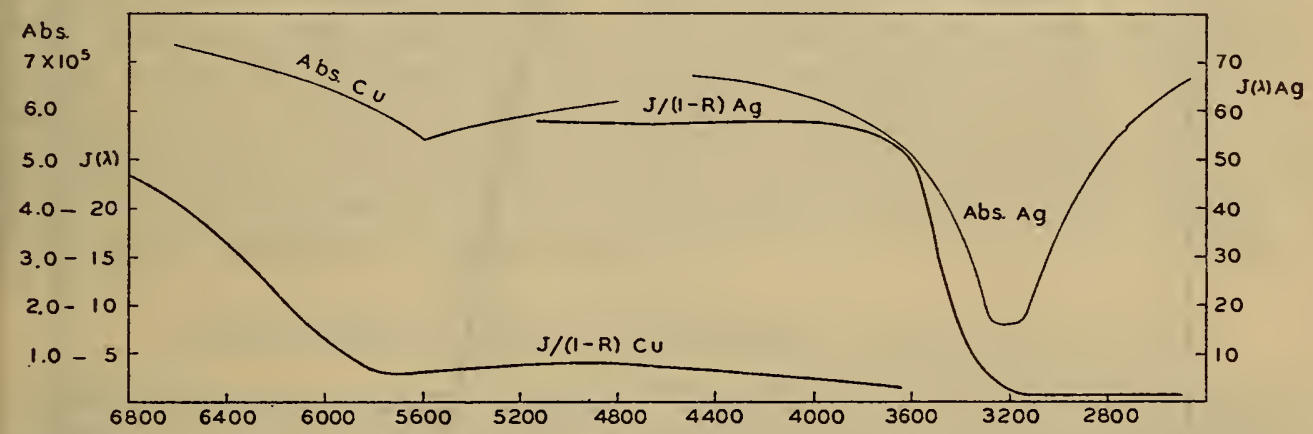

FIGURE 7.-Iniensity distribution of silver and copper radiation corrected for internal reflection in units of Figure 2

Absorption coefficients of copper and silver in $\mathrm{cm}^{-1}$.

trons falls into the vacant place emitting radiation, with the resulting spectrum a continuous band because of the wide range of energies of the free electrons. High dispersion spectra of soft X-ray emission lines show the broadening and Söderman's ${ }^{6}$ densitometer curves of Be $K a$ are the best illustration. This line appears as a band extending from 110 to $125 \mathrm{~A}$ with the maximum near the short-wavelength limit. The selective emission of silver is assumed to be such a band with the long-wave-length limit somewhere in the infra-red. This view is supported by the fact that the short-wave-length part of the band between 3,800 and $3,100 \mathrm{~A}$ is strikingly like the high velocity end of a Fermi distribution in energy as is borne out by the following computation:

The Sommerfeld equation for this distribution is: ${ }^{7}$

$$
F(E)=\frac{c_{1} E^{1 / 2}}{\exp \left(e\left(E-W_{i}\right) / k T\right)+1}
$$

where $E$ is the electron energy, $W_{i}$ the "internal work function," $T$ the absolute temperature, and $e / k=11,600$ for $E-W_{t}$ in volts.

3 Houston, Remarks made in presentation of paper No. 61, Washington Meeting of the Physical Society, May 1,1931 .

6 Söderman, Zeits. f. Phys., vol. 65, p. 656; 1930

7 Darrow, Phys. Rev. Supplement (Rev. of Modern Physics), vol. 1, p. 90; 1929. 
We will apply equation (3) to a range of values of $E$ small in comparison to $E$ and treat $c_{1} E^{1 / 2}$ as a constant, $c_{2}$. Then $F(E)$ is equal to $c_{2} / 2$ for $E=W_{i}$ and it increases to $c_{2}$ and decreases to zero symmetrically for $E$ less or greater than $W_{i}$. Figure 8 shows that the intensity distribution is of the form

$$
\begin{aligned}
J & =\frac{c_{2}}{\exp x+1} \\
x & =e\left(V-V_{0}\right) / k T
\end{aligned}
$$

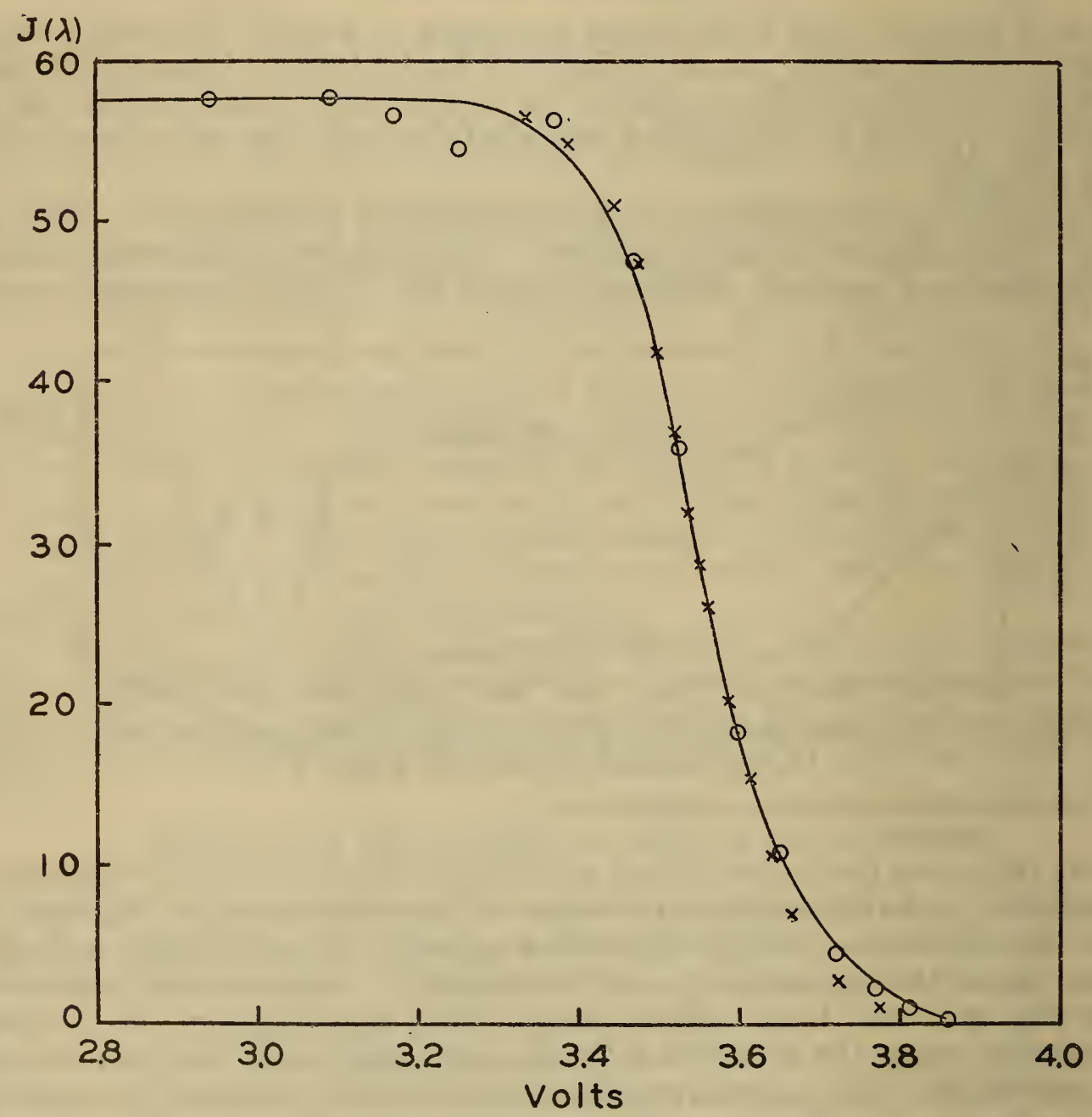

FIGURE 8.-Circles, silver intensity corrected for internal reflection; crosses, Fermi distribution in energy of electrons at $370^{\circ} \mathrm{C}$. on the same scale

with $V_{0}$, the origin of $x$, at 3.56 volts and $x=1$ for $V-V_{0}=0.055$ volts. This gives $T=640^{\circ} \mathrm{K}$ or $370^{\circ} \mathrm{C}$. As the cæsium tube is held at $200^{\circ}$ $\mathrm{C}$. and the probe is considerably heated by electron bombardment the assumed value of $x$ is a reasonable value.

This result supports modern theories of metallic conduction in contrast with classical theories in indicating that the conduction electrons have a wide range of energies with a distribution consistent with equation (3) near the maximum energy. It does not give specific evidence that the entire distribution is like a Fermi distribution. Further $V$ and $V_{0}$ of equation (4) are not identical with $E$ and $W_{t}$ of equation (3) for the origin of $V$ is undetermined. Houston suggested that the underlying level is part of a "Bloch distribution" 
of free electrons in which case $W_{i}$ is greater than $V_{0}=3.56$ volts. However, the above computation indicates that the lower level is sharply defined and not a diffuse band for the entire spread of the edge of the emission band is accounted for by the temperature effect on the upper energy level. This suggests that the lower level is a discrete atomic level and that the energy range of "free" electrons is less than 3.56 volts. The range of a Fermi distribution assuming one electron per atom is 5.6 volts in silver. ${ }^{8}$

Since the continuum of permitted electron levels up to 3.56 volts above the lower level are occupied, the minimum potential required to ionize the lower level and excite any of the radiation is 3.56 volts. The intensity voltage curve for silver (fig. 5) measures the excitation of the selective band and the intercept 1.7 volts gives $V+W=3.8$ volts in fair agreement with the predicted value. Further, the form of the curve is like that of a curve of ionization versus voltage. ${ }^{9}$

Corresponding to electron excitation one would expect to find photoelectric absorption extending from the threshold of the emission band toward shorter wave lengths. The absorption curve of Figure 7 gives evidence of such an absorption band though the short-wavelength edge of the emission and the long-wave-length edge of the absorption are not exactly coincident. However, other features of the absorption curve are not explained and the distinctive feature of silver absorption is the low value near 3,200 A rather than the high value at shorter wave lengths. ${ }^{10}$

\section{THE VARIATION OF INTENSITY WITH VOLTAGE}

There have been many studies of the photo-electric effect of the total radiation from an anticathode bombarded by electrons with energies ranging from 3 or 4 to over 1,000 electron volts. ${ }^{11}$ The current voltage curves, in general, rise with increasing voltage, but with many sharp bends where the slope either increases or decreases. It was originally considered that these bends measured excitation potentials of characteristic X-rays, but it has become evident that known atomic energy levels can not account for all the observed discontinuities. The continuous spectrum observed in our experiments must account for at least part of this effect and it is seen from the voltage intensity curves of Figures 5 and 6 that the integrated photo-electric effect of this continuous spectrum will give sharp inflections in the currentvoltage curve.

It has been shown in the preceding section that characteristic $\mathrm{X}$-radiation of long wave length will give wide continuous bands and not lines and it is evident that, if there were a selective emission like that of silver with its high-frequency limit in the Schumann region and its excitation potential 7 or 8 volts the whole observed spectrum might increase at this potential. This is qualitatively what was observed for aluminum radiation. A sudden increase in intensity began near $V=7$ volts $V+W=9$ volts. The increase extended to beyond $5,000 \mathrm{~A}$, which would require an electron distribution of over 6.5 volts, much greater than silver. But Be, $\mathrm{K} \alpha$ from metallic beryllium

8 Darrow, Phys. Rev. Supplement, vol. 1, p. 122; 1929.

- Franck and Jordan, A nregung von Quanten Sprüngen durch Stösse, p. 183. Julius Springer.

10 Kronig (Proc. Roy. Soc., vol. A 124, p. 409, 1929) accounts for the observed optical properties of silver by transitions of the free electrons to a continuum of levels. A sharp selection principle, however, permits transitions of a given electron to but one of the many possible levels.

i1 Holweck, De la Lumiere aux Rayons X; Univ. of France Press. 
is a band over 12 volts wide, so the lower limit of 6.5 volts is not unreasonable. Sommerfeld's theory gives the range of the Fermi distribution with 1 electron per atom as about 5.6 volts for aluminum as well as silver. However, the above considerations do not explain why the aluminum intensity curve should have a sharp maximum at 11 volts and the evidence for ascribing the peak in the voltage curve to characteristic radiation is not conclusive.

There are other factors which will modify the intensity voltage variation of the continuous spectrum and the integrated photoelectric effect, notably internal scattering of the electrons. If the incident electron penetrates the metal to a depth such that emergent radiation is strongly absorbed, then the occurrence of scattering at some potential may increase the emergent radiation simply by keeping the electrons nearer the metal surface. ${ }^{12}$ Since scattering is probably a difraction process, any potential variation of intensity from this cause will depend on crystal structure and orientation rather than on atomic energy levels. Experiments are in progress to investigate the part played by crystal structure, but at present we can add little to existing evidence for and against a correlation between electron scattering and radiation potentials. ${ }^{13}{ }^{14}$

In conclusion we wish to acknowledge our indebtedness to $\mathrm{Mr}$. Wahler, of this laboratory, who made all the discharge tubes used in this work. The making and mounting of suitable probes of various materials involved many technical problems which he ingeniously solved.

Washington, August 5, 1931.

12 Rudberg, Proc. Roy. Soc., vol. 120, p. 386; 1928, has proposed this explanation, but in recent correspondence he expressses doubt as to whether it is an important factor.

13 Rupp, Natur. Wiss., vol. 18, p. 880; 1930.

11 Richardson, Proc. Roy. Soc., vol. 128, p. 63; 1930. 